\title{
Product Data Management as a Key Component of Integrated Enterprise Information Systems
}

\author{
Dr. J. X. Gao* and Mr. G. Bursell ** \\ *School of Industrial \& Manufacturing Science, Cranfield University, Bedford, MK43 OAL, UK \\ ** Mabey \& Johnson Ltd., Lydney Industrial Estate, Lydney, Glos. GL15 4EJ, UK.
}

\begin{abstract}
Faced with increasingly competitive markets, the need to reduce lead times and the need to react to customer demands more effectively, manufacturing resource planning (MRPII) systems and computer-aided design (CAD) have become commonplace in manufacturing companies. These are, however, implemented in isolated and autonomous locations and without thought to their integration. Where integration is achieved technically, operational, philosophical and organisational issues have often presented an inaccurate and uncoordinated solution resulting in sustained bottlenecks between design, manufacturing planning and production processes. This document presents a case study of a manufacturing company facing these issues in a technology driven manufacturing strategy and proposes a framework to resolve them, based around matured Product Data Management (PDM) technologies. Learning the lessons from earlier direct application integration, the notion that integration of these systems is not only an issue of data but also business processes, as manufacturing companies embrace collaboration with their suppliers, customers and suppliers is also presented. This requires control, visibility and flexibility throughout enterprise information systems.
\end{abstract}

Key words: product data management, PDM, computer-aided design, CAD, manufacturing resource planning, MRPII, integration

\section{INTRODUCTION}

The increasing use of CAD and design automation solutions generates vast amounts of data and documents. The maturing of Product Data Management (PDM) as a means of managing this is becoming increasingly prevelant along with philosophies like Concurrent Engineering in 
manufacturing companies. However, bottlenecks are still being maintained between design and manufacturing with high degrees of redundancy, manual data entry and re-entry, duplication and errors. The role of PDM has changed, today playing a strategic role in removing these bottlenecks and promoting a single integrated solution with manufacturing resource planning (MRPII) systems.

\section{INFORMATION SYSTEMS' INTEGRATION}

\subsection{Design and Manufacturing Information Systems}

The use of CAD at almost all stages of a typical design process is today widespread, from initial concept and specification through analysis and manufacture. Product Data Management (PDM) has evolved to handle the increasingly large amounts of data and complex product and process relationships generated by engineering design automation with $\mathrm{CAD}$ and remove the weakness in manufacturing planning systems of handling master and structural data during early cycles of the design process and even through product development where there are inherent uncertainties. A PDM system is a software tool used to solve manufacturers problems in managing and controlling their engineering data, especially related to new product introduction and engineering processes and, more recently, throughout a product's entire lifecycle [1]. The system comprises three functional categories: the Vault, User functions and Utility functions [2].

Computerised manufacturing planning systems have been in use since the early 1960s, initially as a method of determining and planning material requirements for production. As new tools were added, including production planning, master scheduling, capacity requirements planning and the ability to execute materials and capacity plans, Materials Requirements Planning (MRPII) systems evolved, highly integrated systems optimising materials, procurement and manufacturing processes and providing financial reports. The final evolution, into today's Enterprise Resource Planning (ERP) systems, includes finance, human resources, distribution and traditional accounting functions.

The cornerstone of both of these systems is the bill of material (BOM) and item master records. The BOM, or product structure, documents both part relationships and quantities as well as establishing the logical sequence of events in the manufacturing domain, whist, driven from CAD, it defines the finished physical hierarchical product structure used throughout an enterprise in engineering [3]. The product structure is reliant on the underlying part specification, or item master. From $\mathrm{CAD}$ the item master 
identifies and catalogues drawings whilst in the manufacturing domain it describes and documents what is constantly true about a part. Together, these elements form the basis around which integration revolves, effective only within operational and business processes [4].

\subsection{Models of Integration}

The bill of material or product structures are created and maintained separately in both engineering and manufacturing domains. This often involves a high degree of redundancy, manual data entry, duplication and errors. The scenario of maintaining separate engineering and manufacturing product structure representations has largely been maintained throughout interfacing and integration efforts [5], even though widely adopted philosophies such as Concurrent Engineering promote collaboration between design and manufacturing departments, processes and people in delivering tangible benefits. The co-existence of PDM and MRPII systems is a widely held key requirement in enterprise-wide requirements for manufacturing companies [6] however their integration provides an extensive range of benefits.

Whilst CAD integration with PDM systems is today classed as a commodity, there are a number of considerations in integrating PDM with MRPII systems [7]. Issues such as two master databases and data ownership contention are commonplace. Three typical models of integration can be identified [8] [9], either system becoming a single master data source or, as is more commonly found, item master and product structure information simultaneously exists in both systems, each mutually updating the other through formal engineering change mechanisms. Manufacturing systems are not well suited to the latter as access control mechanisms are required to progagate changes real-time. Where this has been achieved, any interruption in communication with the PDM system has the potential for BOM updates not being fed into manufacturing. The favoured approach is to create and modify BOMs in the PDM domain and feed them into manufacturing through change orders [10], with process workflow particularly well suited to this approach. More often, batch updates propagate changes over night, yet this does little for companies who require regular MRP runs and who would have inaccurate data. Rather, integration bespoke to each company's requirements. The methods of connectivity between PDM and MRPII systems are shown in Figure 1, showing both traditional dependency and abstraction, which have been greatly effected by recent technology developments. In bridging legacy MRPII systems, a gateway application is often favoured. 


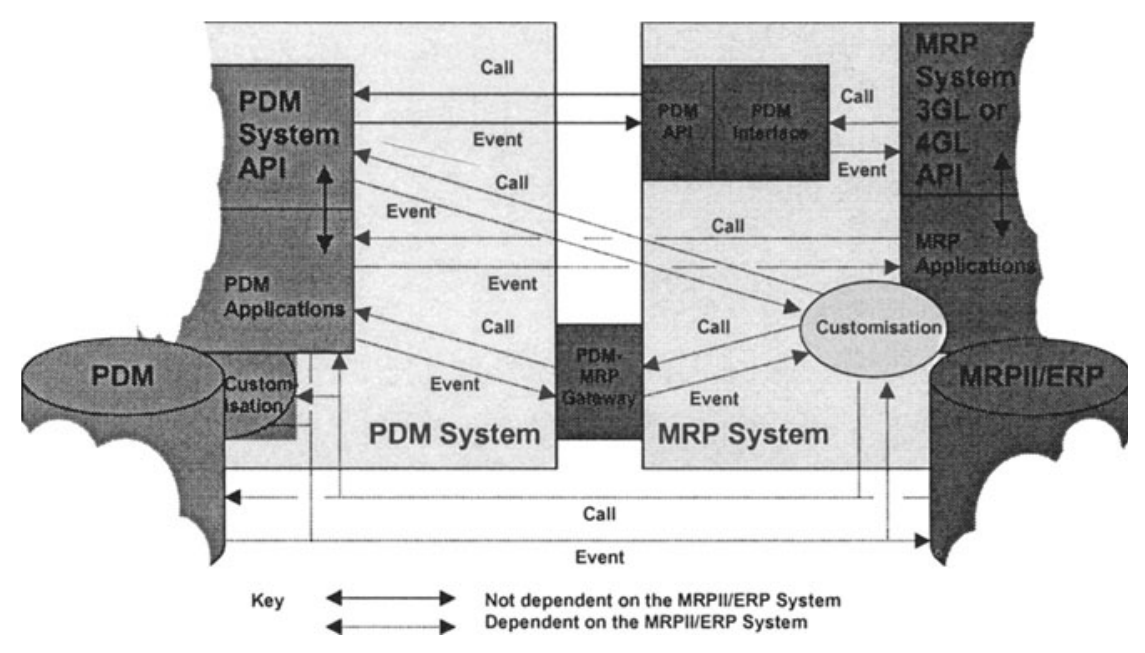

Figure 1. Integration connectivity between PDM and MRPII.

\subsection{Contemporary Developments}

There are encouraging signs that advances in information technology and related standards are finally delivering integration solutions. Both PDM and MRPII systems are embracing object-orientation, on top of operating system platform with object-based application programming interfaces, such as Microsoft's Component Object Model (COM) and ActiveX interfaces through to object integration frameworks like CORBA and distributed objects such as DCOM. The traditional restrictions of rebuilding interfaces when system configurations change or upgrades are installed are being replaced by integration techniques of providing a layer of abstraction outside of the core systems with integration specific APIs which provide high flexibility with low overheads [11].

However, many manufacturing companies maintain legacy secondgeneration MRPII systems, with closed 3GL APIs. Database standards such as SQL are removing the restrictions of application integration with these systems, where supported, yet the real developments in recent times have come with platform and system independent standards like Standard for the Exchange of Product Data (STEP) and the explosion of e-commerce with hypertext markup language (HTTP) and Extensible Markup Language (XML) [12] being exploited for integration. The XML specification has elicited support from many MRPII, ERP and PDM vendors with many such systems providing license free and platform independent 'connectors' [13] to major integration partner systems like SAP, Matrix, Baan and Sherpa. XML 
heads the re-emergence of neutral file formats for integration, applying a messaging protocol, which encapsulates structured data and metadata as well as content based on data model definitions, or schemas, held in Microsoft's Biztalk or RossettaNet frameworks. In addition, Biztalk Server provides messaging, and workflow capabilities to expand existing applications and information systems beyond their existing boundaries.

Further developments of note are the issues of scaleability in implementing PDM systems, often to world-wide locations. The development of standards and communications protocols today makes issues of platform dependence redundant.

\section{CASE STUDY}

\subsection{Company Background}

Mabey \& Johnson Ltd is a privately owned company specialising in the design and manufacture of modular prefabricated steel bridging systems, which are supplied primarily from their facilities in Lydney, Gloucestershire to customers worldwide. Modular bridging systems are used in a variety of applications including temporary, permanent, floating and emergency bridging requirements. They are easy to transport and can be erected with unskilled labour with minimal engineering plant. Some $90 \%$ of the company's production is exported to over 130 countries, either ex-stock or to the client's own specification in a rapidly growing world market. This is especially prevalent in developing countries and those emerging from armed conflict that are expanding or repairing their transportation infrastructures.

The company has invested heavily into highly automated manufacturing technologies from which production throughput is predominantly in the form of standard products, fabricated by robots. These make up $80 \%$ of all production; the remainder being engineered to order, which is, processed through manual welding facilities and the use of sub contract manufacturers. The manual welding and fabrication facilities are maintained to accommodate non-standard requirements and to supplement the automated fabrication processes. As well as sales of bridging systems, a certain stock level for hire is maintained enabling the company to quickly react to requirements. Products with long manufacturing lead times are held as finished stock and demand is often volatile and highly uncertain.

Identifying rapid customer respfonse, high quality and flexibility as critical business success factors, the company commenced a rolling investment program in 1995 in the development of an enterprise-wide Management Information System (MIS), which is now nearing completion 
[14]. This combines state-of-the-art manufacturing and information technology to reduce design and manufacturing lead times, including a reliance on $3 \mathrm{D}$ solid modelling $\mathrm{CAD}$ and Computer-Aided Engineering (CAE) tools. In a technology driven manufacturing strategy, the provision of an Engineering Support System (ESS) framework to centralise and coordinate activities and information between the various departments, processes and computerised engineering and manufacturing systems has been identified as the key facilitator to realise the full benefits of already highly effective islands of automation.

\subsection{Proposed Engineering Support System framework}

The proposed Engineering Support System (ESS) framework described is the result of an investigation into the availability of suitable information technology solutions, integration standards and technologies and operational business requirements. The purpose of this framework is to directly bridge support applications to the manufacturing planning and production domains and provide the foundation of computer-aided manufacture (CAM) integration, areas that were not considered in the development of the specialised hybrid planning framework implemented [14]. During this period a vast array of technologies, standards, suppliers and vendors were systematically reduced to a shortlist, however no integral solution existed and, as a consequence, a high degree of customisation and application development exists in all scenarios to present a solution. As a consequence, the ESS framework is constructed in four parts, supporting the operational focus of the company highlighted in the planning framework, material management and manufacturing execution by providing core level data and document control, process control, product structure management and engineering and manufacturing data and process integration. The foundation of the ESS framework is a PDM system, positioned as a layer around the manufacturing planning system to control the flow of information both in and out of this domain. An overview of the ESS framework is shown in Figure 2 and is described in subsequent sections. 


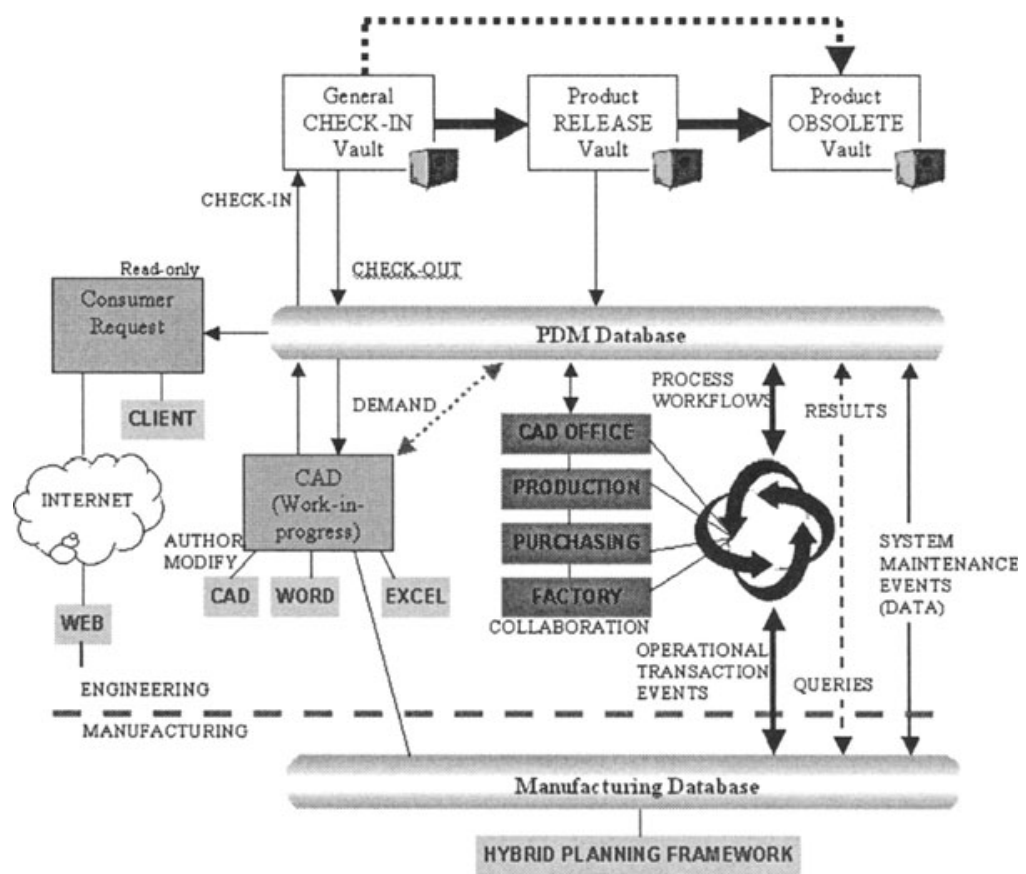

Figure 2. Proposed Engineering Support System framework.

\subsection{Data and Document Control}

The ESS framework will provide a coordinated engineering package of associated documents and data generated throughout a products entire life cycle. Document and data classification will provide two categories, identifying documents and data used directly in production, such as fabrication drawings, and associated supporting documents and data, such as analysis results from produced early in the product design process. All documents are revision controlled, with all changes tracked and the complex relationships and dependencies that exist managed to enable the engineering data packaging. This package would then be released to production.

Document storage is within a centralised vault, with security-controlled access. This is an important issue, as considerable reworking of CAD models has been found due to a lack of control. A multiple vault topology will be utilised, separating released documents and data from checked-in work in progress and obsolete storage vaults. This will also provide the archiving requirements for ISO 9000 quality standards compliance. The engineering package is moved to the released vault upon approval, however, should any element of the package require revision, the entire package contents are moved back to the checked-in vault as associations and 
dependencies will be maintained across multiple vaults for control. This also means an impact analysis can be performed prior to changes being effected. The accessibility of the engineering packages is dependent on the roles of system users within the ESS environment. For instance, shop floor operators will have print (read-only) capabilities whereas CAD operators will have creation, modify and obsolescence capabilities.

Extensive search and retrieval capabilities, impacting throughout the ESS framework will vastly reduce the time taken to find information and documents in a centralised information repository as well as reducing duplication and redundancy in part creation. A plethora of uncoordinated hardcopy, computerised databases and manual storage mechanisms are currently in use. Automated coordinated part creation will be implemented within the ESS framework.

\subsection{Process Control}

The engine of the ESS framework is PDM-based workflow. This will be used to initiate, manage and track business processes; primarily approved release to manufacture, distribution and engineering change control. Processes will be modelled graphically within the PDM system in a flow chart format. The workflow definition is held within the PDM data model, allowing process flows to be adapted on the fly to changing requirements without the need to recompile a process before it can be used. Workflow augments business processes by packaging and routing associated data and documents between various stages, via approval or rejection, through departments and people in the enterprise. Traditional paper documents, such as engineering change request forms, will be amalgamated as data within an engineering change process workflow, collected or input through on-screen forms, matching company documentation and procedures. Workflow is not a linear process; indeed iterative, concurrent and serial activities will be found in almost all business processes. Adopting workflow as the core engine of the ESS framework will remove superfluous steps and inefficient activities in existing processes, as this will negate many of the benefits of this integration.

Visibility will be raised within business and operational processes, allowing workflows to be tracked, audited and archived and provide complete traceability in engineering as well as in manufacturing. Complex processes will also be handled as process workflows can also be nested and contain rule-based event triggers (decisions) to improve routing speed in a controlled manner. Manual processes can also be recorded and escalation policies will be enforced to ensure processes such as drawing approval, a problematic bottleneck, are completed. Combining drawing and 
manufacturing job information, such as planned start date, within drawing approval workflows, for instance, will enable problematic processes to be prioritised and, if necessary, escalated, to ensure all engineering information is released by the time production commences. User notification, found at various stages of workflows, is integrated with electronic mail messages and provides feedback and action requirements to users involved in workflows.

The role of workflow and process control in the ESS framework environment will ensure that if something needs to happen, it happens by the time it is needed. Conversely, if a process is not completed on time, visibility highlights who or what the bottleneck is. Engineering data, often dynamic in nature, is of no use to manufacturing if it does not arrive on time, yet in contrast; manufacturing data is relatively static and defined in detail. This fact underlines why processes must be managed to be effective. Errors, duplication, redundancy and lead times can all be reduced, yet a coordinated and centralised engineering package available to manufacturing will facilitate the high levels of flexibility required in manufacturing through adopted workflow processes.

\subsection{Product Structure Management}

The Configuration Management (CM) aspect of PDM in the ESS will manage two revision controlled product structure (BOM) views, or asdesigned and as-planned configurations. The creation of product structures is exclusively performed in the PDM domain, utilising synchronised item masters from the manufacturing system. This is to enable the traditional push of data from engineering into manufacturing and is enforced as a series of business rules within operational process workflows. The development of the manufacturing product structure (MBOM), incorporating process routes, evolves from the intrinsically captured $\mathrm{CAD}$-based engineering product structure (EBOM) far earlier in the design process, coupled with $\mathrm{CM}$ to manage changes that occur over time. Collaboration at an early stage will allow purchasing and planning functions to happen more as a consequence than as a demand and will allow impact analyses of engineering change requests to be preformed.

Product structures are built graphically, providing greater adaptive flexibility and visibility and are consolidated to provide the enterprise-wide product structure definition, although multiple configurations will inevitably exist in product development. The consolidation of engineering and manufacturing product structures is shown in Figure 3, providing coordinated effectivity controls for engineering change requests and orders. 


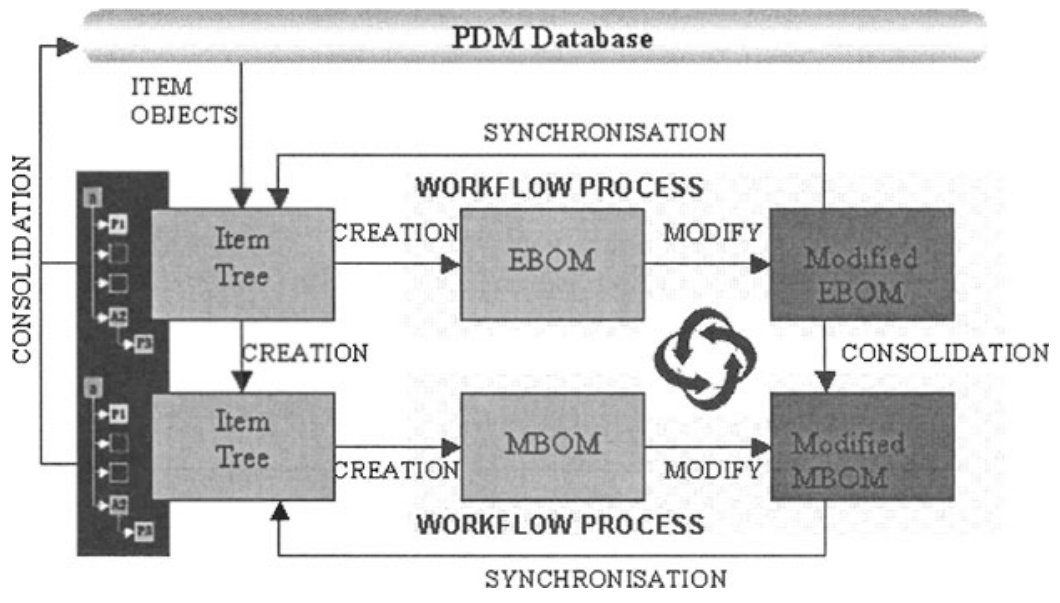

Figure 3. Engineering and Manufacturing BOM creation and control.

Manufacturing planning functions such as rough-cut capacity planning and procurement are significantly improved as a completed product structure is generated through the workflow transport in a distributed collaboration.

\subsection{Data and Process Integration}

Engineering and manufacturing data and process integration will take place at three levels between the PDM and manufacturing systems, however a feature of the ESS framework is that no direct integration or tethering of the two core systems takes place, rather a bi-directional messaging mechanism is implemented. Both systems are regarded as mutually controlling masters in the integration, driven through workflow processes. Both domains will work independently of each other, maintaining their own local data sources, utilising workflow processes to drive interaction.

While no workflow or CM capabilities exist in the manufacturing system domain, interaction between the two systems is implemented through XML messaging, encapsulating not only messaging content, but also metadata describing the content, structure, definition and context. Using Microsoft's Biztalk Server platform, integrated with PDM workflow, request messages are mapped to actions in one system, redirected through the Biztalk framework and received back as response messages in the second. Examples of this include a request for a completed and approved drawing from the manufacturing system following changes to the master production schedule to begin production and effect changes or create new component and assembly items. This would activate a process workflow in the ESS to 
progress the request and return the response with associated data. In order for this to be an effective approach, business data models and processes will require a complete definition but only need to be implemented once.

The three levels of messaging integration identified are:

1. Process Integration. Also described as operational transactional events, moves multi-part data flow processes between each system. Examples include engineering change requests and part identification. Message information is extracted and mapped to workflow nodes. This will be implemented by API integration between Biztalk Server and PDM workflow to transparently extend process workflows outside the PDM system and into the enterprise. The data requirements of these processes are held in schemas. Changes to a business process will therefore only require a change to the schema definition and routing of the process, as opposed to additional API integration. Irrespective of the computing platforms, Biztalk hands off messages to both systems but this is reliant on business processes being explicitly defined in both systems.

Specific processes, such as engineering change, require external coordination. This will be performed by a user with detailed product and process knowledge as well as who utilises both systems and will involve dynamically re-routing workflow processes, reviewing and tracking process status and making strategic decisions to maintain optimum efficiency of the integrated systems' functions. Process integration will also allow engineering demand to be presented and scheduled outside of the manufacturing system to meet demand and production commencement lead times, especially following changes to the master production schedule in the manufacturing planning system.

2. Data Integration. In addition to triggered process flow events, the positioning of two perceived mutual master data sources requires maintenance transactions for synchronisation with transaction logging.

3. Query Integration. Principle information consumption in all areas will be through a web browser interface, where manufacturing job-related information, such as works instructions and process plans, is associated with the engineering package. This level of presenting ad-hoc read-only information from the manufacturing domain will be implemented through a COM OLE-DB service provider, implemented through visual basic API scripting on demand for fast data retrieval.

Non value-added functions are removed to reduced server-side processing, plying business processes and data to perform integrated functions as a consequence of operational processes, not in spite of them as has traditionally been the effect of integration. 


\section{CONCLUSIONS}

In order to achieve the required levels of integration between PDM and MRPII systems, both core data and documents as well as business processes must be effectively managed together. The use of PDM systems and independent standards now affords companies with legacy MRPII systems the same benefits today's leading edge ERP systems provide.

\section{REFERENCES}

1. KIRBY, H. PDM - What do you need to know? Works Management, Vol. 52(5), 1999, 25-28.

2. CIMdata INC. Product Data Management: The Definition. An Introduction to the Concepts, Benefits and Terminology. CIMdata Inc., 1996.

3. FrANK, D. N. The Business Case for Interoperability. APICS, Vol. 12(5), 1999, http://www.apics.org/magazine/May99/interoperability.htm

4. RUSK, P. S. The role of bill of material in manufacturing systems. Engineering Costs and Production Economics, Vol. 19(1-3), May 1990, 205-211.

5. HASIN, M. A. A. AND PANDEY, P.C. Analysis of present scheme of MRPII-CAD integration: problems and prospects. International Journal of Computer Applications in Technology, Vol. 9(23), 1996, 126-130.

6. BARTUli, G. AND BoURKE, R. W. The Best of Both Worlds: Planning For Effective Coexistence of Product Data Management and MRP II Systems. February 1995, http://www.pdmic.com/articles/bestbth1.html, http://www.pdmic.com/articles/bestbth2.html

7. GREGORY, A. Linking PDM systems to manufacturing. Manufacturing Computer Solutions, Vol. 4(4), April 1998, 34-36.

8. Miller, E. PDM/ERP REPORT: Integrating PDM and ERP. Computer-Aided Engineering, March 1999, http://www.caenet.com/res/archives/9903pdmerp.html

9. CANAL, B. The PDM - MRP Link. Proceedings of the Technical Program. NEPCON East '95. On Target with Today's Technology and Tomorrow's Challenges, 12-15 June 1995, Boston, USA, 8492.

10. HALL, G. PDM: Integration challenge. CADCAM, Vol. 17(7), July 1998, 27-28.

11. FERMAN, J. E. Strategies for successful ERP connections: Techniques and their technologies for integrating systems. Manufacturing Engineering, Vol. 124(4), 1999, 48-60.

12. GouldE, M. A. Microsoft's Biztalk Framework adds messaging to XML. Proposed framework for XML schemas and exchange of data. E-Business Strategies and Solutions. September 1999, 10-14.

13. MAINWARING, J. EAI - sticking it all together. Manufacturing Computer Solutions, Vol. 6(2), 2000, 28-30.

14. Beach, R, Muhlemann, A. P, Price, D. H. R, Paterson, A. and Sharp, J. A. Information systems as a key facilitator of manufacturing flexibility: a documented application. Production Planning \& Control, Vol. 9(1), 1998, 96-105. 